\title{
Antidiarrheal Agent
}

National Cancer Institute

\section{Source}

National Cancer Institute. Antidiarrheal Agent. NCI Thesaurus. Code C266.

A pharmacological agent that may ameliorate the symptoms of diarrhetic illnesses. 